\title{
OCUPACIONES HUMANAS DEL HOLOCENO TARDÍO EN LOS VILOS (IV REGIÓN, CHILE): ORIGEN Y CARACTERÍSTICAS CONDUCTUALES DE LA POBLACIÓN LOCAL DE CAZADORES RECOLECTORES DE LITORAL
}

\author{
LATE HOLOCENE HUMAN OCCUPATIONS IN LOS VILOS \\ (REGION IV, CHILE): ORIGIN AND BEHAVIORAL CHARACTERISTICS \\ OF LOCAL COASTAL HUNTER GATHERERS
}

César A. Méndez. M.* y Donald G. Jackson S. **

\begin{abstract}
Se presenta una propuesta interpretativa al registro arqueológico generado por grupos de recolectores cazadores durante el Holoceno tardío ( 4.000 y 2.000 años cal. a.p.) en la Comuna de Los Vilos (IV Región, Chile). Se propone un modo particular de aproximación al medio litoral por parte de grupos humanos que habitaron la zona vinculado a la apropiación de alimentos a nivel diario y la movilidad residencial en función de la disponibilidad de los recursos. Se plantea la factibilidad de discriminarlos analíticamente de poblaciones más nortinas, junto con establecer vínculos más certeros con los desarrollos culturales meridionales.
\end{abstract}

Palabras claves: Holoceno tardío, cazadores recolectores, variables conductuales, litoral semiárido de Chile.

We present an interpretative model for the late Holocene hunter-gatherer archaeological record at Los Vilos (Region IV, Chile). We suggest that the human groups that inhabited this region between ca. 4,000 and 2,000 cal. B.P. had a specific manner of utilizing the littoral environment. The proposed model, based on hypothesized daily food procurement rounds, takes into account residential mobility as well as resource distribution. Our analysis suggests that there were significant differences between populations of this zone and those further to the north, while at the same time demonstrating closer connections with groups existing further to the south.

Key words: Late Holocene, hunter gatherers, behavioral variables, semi-arid coastal region of Chile.

Los desarrollos culturales de cazadores recolectores del Holoceno tardío permiten frecuentemente observar interesantes procesos de larga duración, en donde el hombre ha generado estrechos vínculos con el ambiente. La comuna litoral de Los Vilos (Provincia del Choapa, IV Región) no es ajena a dicha realidad, en vistas que en la actualidad es posible observar una completa secuencia cronológica para aquel segmento denominado Arcaico. El objetivo principal de esta comunicación es exponer un modelo interpretativo para los contextos generados por estas poblaciones. Este se sustenta sobre la exploración superficial sistemática y ubicación de sitios arqueológicos, en conjunto con un programa de sondeos y la intervención estratigráfica de algunos yacimientos. En esta instancia, se pretende dar cuenta de las características contextuales de los sitios estudiados, y discutir la organización tecnológica, estrategias de subsistencia y patrones de movilidad de estas poblaciones.
Hacia 1995, el registro arqueológico de los cazadores recolectores del Holoceno tardío en Los Vilos se caracterizaba como ocupaciones que

adquieren un carácter intensivo, que se manifiesta en depósitos densos compuestos por una gran variedad de moluscos. Incluyen tanto bivalvos como gastrópodos, estos últimos frecuentemente fracturados, asociados a erizos y a varias especies de crustáceos. Reflejan una explotación intensiva de la biodiversidad malacológica del sistema intermareal (Jackson et al. 1995:108).

Si bien la descripción expuesta revela un claro patrón contextual, que difícilmente podría ser modificado en la actualidad, el entendimiento de las tendencias conductuales y culturales prevalecientes durante este lapso no se encuentra del todo

\footnotetext{
* Universidad Católica del Norte; cmendezm@uchile.cl

** Departamento de Antropología, Facultad de Ciencias Sociales, Universidad de Chile. Ignacio Carrera Pinto 1045, Ñuñoa, Santiago; djackson@uchile.cl
} 
resuelto. La comprensión de las dinámicas de los cazadores recolectores, a la luz de planteamientos teóricos explícitos y las nuevas evidencias aquí presentadas, permite desarrollar tanto un referente explicativo de mayor claridad como novedosas hipótesis regionales.

\section{Ambiente}

El Holoceno tardío se caracteriza, en términos generales, por el advenimiento de condiciones climáticas más húmedas y frías que aquellas inmediatamente anteriores. La zona de Los Vilos constituye, en este sentido, un área privilegiada, puesto que se han conducido importantes estudios para dilucidar su ambiente pretérito (Maldonado y Villagrán 2002; Núñez et al. 1994; Villagrán y Varela 1990). En la zona III (miembros 5 y 6), de la columna palinológica de Quereo, hacia los 3.200 años cal. a.p., se exhibe la recolonización de especies pantanosas (de origen acuático), la cual daría cuenta de la presencia de vegetación semiárida más variada en condiciones húmedas (Núñez et al. 1994; Villagrán y Varela 1990). Recientes investigaciones corroboraron la recolonización arbórea, fechándola hacia los 4.200 años cal. a.p., con posteriores fluctuaciones menores que devinieron en el clima actual (Maldonado y Villagrán 2002).

En el ámbito litoral, el ambiente se encuentra significativamente influenciado por el medio marino. Durante el Holoceno, su nivel cambió, pudiéndose establecer posterior a los 4.000 años cal. a.p. el inicio de una serie de fluctuaciones menores hacia su posición actual (Ota y Paskoff 1993). Estas fluctuaciones pueden ser vinculadas a eventos climáticos denominados como Neoglaciales, o tiempos caracterizados por una menor temperatura y mayor pluviosidad (Jerardino 1995). Investigaciones en sectores más meridionales (3330’ S) han permitido corroborar, hacia los 3.800 años cal. a.p. temperaturas marinas más frías, para que posteriormente se iniciase un alza moderada, en fechas del orden de los 2.000 años cal. a.p. (Falabella et al. 1991).

En términos geomorfológicos, se distinguen, en el área de estudio, las siguientes unidades: un sistema de terrazas marinas, de altura y momento de formación variables; un sistema de paleodunas (en algunos casos removilizadas por acción eólica); y en el sector de la desembocadura de Quereo, un graben o cuenca paleolacustre (Vare- la 1981). En su gran mayoría, los contextos estudiados se encuentran emplazados sobre una terraza marina intermedia, entre 25 a $40 \mathrm{msm}$, y sobre depósitos de paleodunas dispuestos sobre el techo de las mismas.

Actualmente, las condiciones climáticas de Los Vilos son de estepa con nubosidad abundante (BSn, Fuenzalida 1965), con precipitaciones anuales promedio de $262 \mathrm{~mm}$, una temperatura media de $14{ }^{\circ} \mathrm{C}$ y se caracteriza por significativa humedad relativa, que fluctúa, según estaciones del año, entre el $70 \%$ y $85 \%$ (Varela 1981). Su vegetación es de estepa o matorral costero, de arbustos y hierbas mesófitas, extendiéndose en el litoral costero entre los $31^{\circ}$ y $34^{\circ} \mathrm{S}$ (Donoso 1981). Propiamente, en el litoral de Los Vilos se registran las siguientes variedades arbustivas: chamucilla (Bahia ambrosioides), baturro (Baccharis concava) y puya (Puya venusta); y herbáceas: picún (Anasomeria litoralis), tupa (Lobelis polypilla) y coralito (Fuchsia lycoides), mientras que en las planicies litorales dominan el palo negro (Haploppapus foliosus), las cactáceas (Eulychnia costanea) y las variedades espinosas (Seguel et al. 1995).

\section{Contextos Arqueológicos Estudiados}

Aunque los contextos y registros tratados a continuación fueron recuperados a lo largo de un lapso prolongado de tiempo, es posible distinguir similitudes en cuanto a su metodología de obtención. Los yacimientos fueron intervenidos subsuperficialmente por niveles artificiales de $5010 \mathrm{~cm}$, en consideración con las unidades depositacionales litoestratigráficas (antrópicas, naturales o ambas). Las dimensiones de las unidades de excavación (y porcentajes de sitio) son variables, por lo que las comparaciones serán atendiendo valores proporcionales, privilegiando su valor cualitativo. Los rasgos fueron debidamente segregados y recuperados independientemente. Asimismo, se llevó a cabo el registro gráfico (dibujos y fotografías) de los perfiles y plantas de excavación. Los sedimentos recuperados fueron íntegramente tamizados en mallas menores a $4 \mathrm{~mm}$, predominando las de 2,5 mm. Complementariamente, se llevó a cabo un sistema de muestreos de flotación y tamizado fino, con el propósito de evaluar evidencias de menor tamaño y el registro de partículas orgánicas imperceptibles en el trabajo en terreno. Las muestras zooarqueológicas (vertebrados e invertebrados) 
fueron identificadas -en lo posible- a nivel específico, a través de su comparación con colecciones actuales. Los materiales líticos fueron evaluados sobre la base de una clasificación morfofuncional (Bate 1971). Finalmente, se dirigió un muestreo sistemático de la talla del loco (Concholepas concholepas), invertebrado que ha demostrado tendencias culturales selectivas en cuanto a su tamaño (Jackson et al. 1996; Jackson et al. 2004; Méndez 2002a).

Para los efectos de este trabajo, se tomó en consideración fundamentalmente contextos con asignación cronológica absoluta. Para ello se fechó muestras por análisis radiocarbónico convencional y AMS, y muestras de rocas quemadas con contenido de cuarzo, para su datación por termoluminiscencia (Feathers 1996; Román y Jackson 1998). Los resultados exponen un segmento bastante ajustado en relación a la secuencia local (Méndez 2002a), mostrando ocupaciones entre los 3.800 y 2.000 años cal. a.p. (calibrados a dos sigma con el programa Ox Cal vers. 3.5, con información ambiental de Stuiver et al. 1998; Tabla 1, Figura 1).

Los siete yacimientos intervenidos corresponden a muestras selectivas, representativas de sistemas de asentamiento de mayor tamaño. De norte a sur, aparece Cabo Tablas-Ñagué (LV. 204, Figura 2), sitio de difícil interpretación; el cual, quizás con la combinación de los depósitos conchíferos adyacentes (LV. 203), permite entender un contexto integrado de mayor coherencia (Artigas 2003). El yacimiento se ubica sobre la segunda terraza marina, adyacente a un talud abrupto que se enfrenta a una playa de guijarros de formación durante el
Holoceno tardío. Se llevó a cabo una extensa recolección superficial, fundamentalmente de material lítico, en conjunto con un sistema de muestreos estratigráficos $\left(6 \mathrm{~m}^{2}\right)$, los que, aunque no exentos de discusión, exhiben dos niveles ocupacionales (Artigas 2003). El principal, de cazadores recolectores del Holoceno tardío, se encuentra casi en su totalidad en superficie. En este sentido, el sitio podía caracterizarse a modo de área significativamente afectada por deflación eólica, cuya remoción de sedimentos constituye un agente generador de un palimpsesto, que coadunó evidencias de distintas ocupaciones humanas (Schiffer 1996). Asimismo, la escasez de restos orgánicos bien podría ser asimilable a aquello expuesto por Borrero et al. (1998-9), en donde la erosión eólica estaría jugando un rol fundamental en la perturbación de los contextos, transformándolos únicamente en distribuciones líticas superficiales, en especial ante la meteorización del registro óseo.

El material lítico se caracteriza fundamentalmente por su expeditividad (Artigas 2003), conducta tecnológica que establece que sus creadores anticiparon la presencia de materias primas ante necesidades de uso (Nelson 1991). Dicha anticipación se manifiesta en la selección del área de aprovisionamiento secundaria en la playa de guijarros adyacente al sitio. La labor tecnológica es simple, y caracterizada por extracciones corticales sin preparación, escasos núcleos e instrumental sobre núcleos y guijarros. No obstante, también fueron registrados escasos desechos de retoque bifacial y un fragmento de cabezal lítico de un sílice de origen foráneo.

Tabla 1. Dataciones absolutas (resultados medidos) de contextos de cazadores recolectores del Holoceno tardío. Absolute dating (average ages) of late Holocene hunter gatherer contexts.

\begin{tabular}{lcrllll}
\hline Contextos & $\begin{array}{c}\text { Fechas } \\
\text { a.p. }\end{array}$ & Ds & Laboratorio & Muestra & Método & Referencia \\
\hline Punta Chungo B & 3.390 & 70 & Beta-121449 & Concha & 14C (conv) & Jackson y Méndez 2004 \\
P. Inf. Conchalí & 3.280 & 50 & Beta-143140 & Concha & 14C (conv) & Barrera y Belmar 2000 \\
Cabo Tablas-Ñagué & 3.160 & 60 & Beta-137025 & Carbón & AMS & Artigas 2003 \\
Punta Chungo A & 2.770 & 60 & Beta-95075 & Concha & 14C (conv) & Jackson et al. 2004 \\
Quereo & 2.475 & 90 & SI-3390 & Carbón & 14C (conv) & Núñez et al. 1994 \\
Quereo & 2.420 & 100 & N-2961 & Carbón & 14C (conv) & Núñez et al. 1994 \\
Quereo Norte & 2.290 & 50 & Beta-105213 & Concha & AMS & Jackson et al. 1998 \\
Quereo Norte & 2.210 & 50 & NSRL-11083 & Carbón & AMS & Jackson et al. 2001 \\
Punta Penitente & 2.160 & 100 & UCTL-1338 & Roca & TL & Méndez 2002b \\
\hline
\end{tabular}




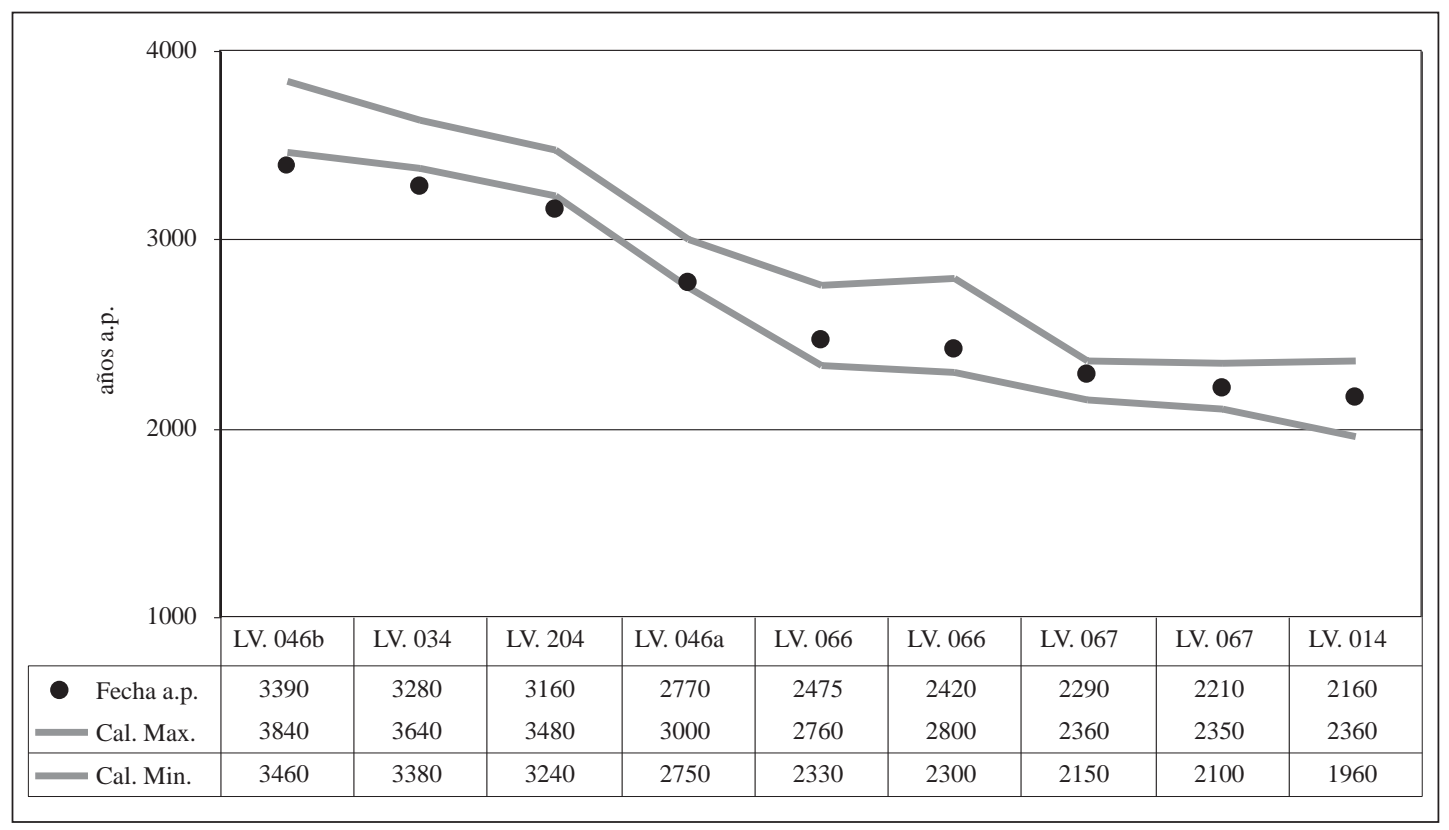

Figura 1. Dataciones calibradas de contextos de cazadores recolectores del Holoceno tardío (salvo datación de termoluminiscencia de LV. 014).

Calibrated dates from late Holocene hunter gatherer contexts (excluding thermoluminiscence dating of LV. 014).

El segundo contexto, Punta Penitente (LV. 014), a $40 \mathrm{msm}$, alude a una funcionalidad residencial de actividades con múltiples propósitos. $\mathrm{Su}$ intervención (muestreos superficiales, análisis de materiales in situ y excavaciones de $4 \mathrm{~m}^{2}$ ) permitió discriminar una potente ocupación de cazadores recolectores del Holoceno tardío, con tecnología de escaso nivel de conservación (Shott 1996), que anticipaba recursos líticos inmediatos (Nelson 1991), evidentes, incluso, en un taller lítico en el mismo sitio (Méndez 2002a). En el contexto se registra una importante actividad de procesamiento malacológico, el cual incluiría extracción de la porción comestible y procesamiento inicial mediante exposición al fuego (sedimentos carbonosos, partículas de carbón y algunos moluscos y rocas con marcas de fuego). Se observó una alta tasa de descarte, constituyéndose una matriz depositacional antrópico/natural, cuyas valvas de invertebrados, profusamente fragmentadas (pisoteo), podrían ser concebidas como partículas sedimentarias (Stein 1992). En cuanto a los restos de vertebrados, se observó evidencias del procesamiento y consumo de aves, roedores, cánidos y ungulados.

Las evidencias líticas permitieron identificar una secuencia de reducción completa, desde la ex- tracción de las formas base (núcleos), matrices, subproductos descartados, desechos de manufactura, hasta el instrumental astillado. Esta tecnología se implementó a fin de llevar a cabo una serie de actividades de procesamiento, posiblemente vinculadas al trabajo sobre maderas, cueros, hueso y moluscos, descartándose en los mismos basurales de valvas. Esta conducta produjo una superposición entre áreas de basurales y habitacionales, conglomerando múltiples evidencias (Méndez 2002a).

Más al sur, otro campamento asignable a estos cazadores recolectores constituye Paso Inferior Conchalí (LV. 034), asentamiento sobre una terraza labrada, adyacente al curso del estero y laguna homónimos.

Las características generales de esta ocupación detectada en la capa $\mathrm{C}$ permiten interpretarla en términos funcionales como un área de desconche y probable consumo de moluscos obtenidos localmente, a la cual se asocia una actividad lítica expeditiva utilizando principalmente materias primas de disponibilidad local e inmediata desde los alrededores del sitio (Barrera y Belmar 2000:2). 


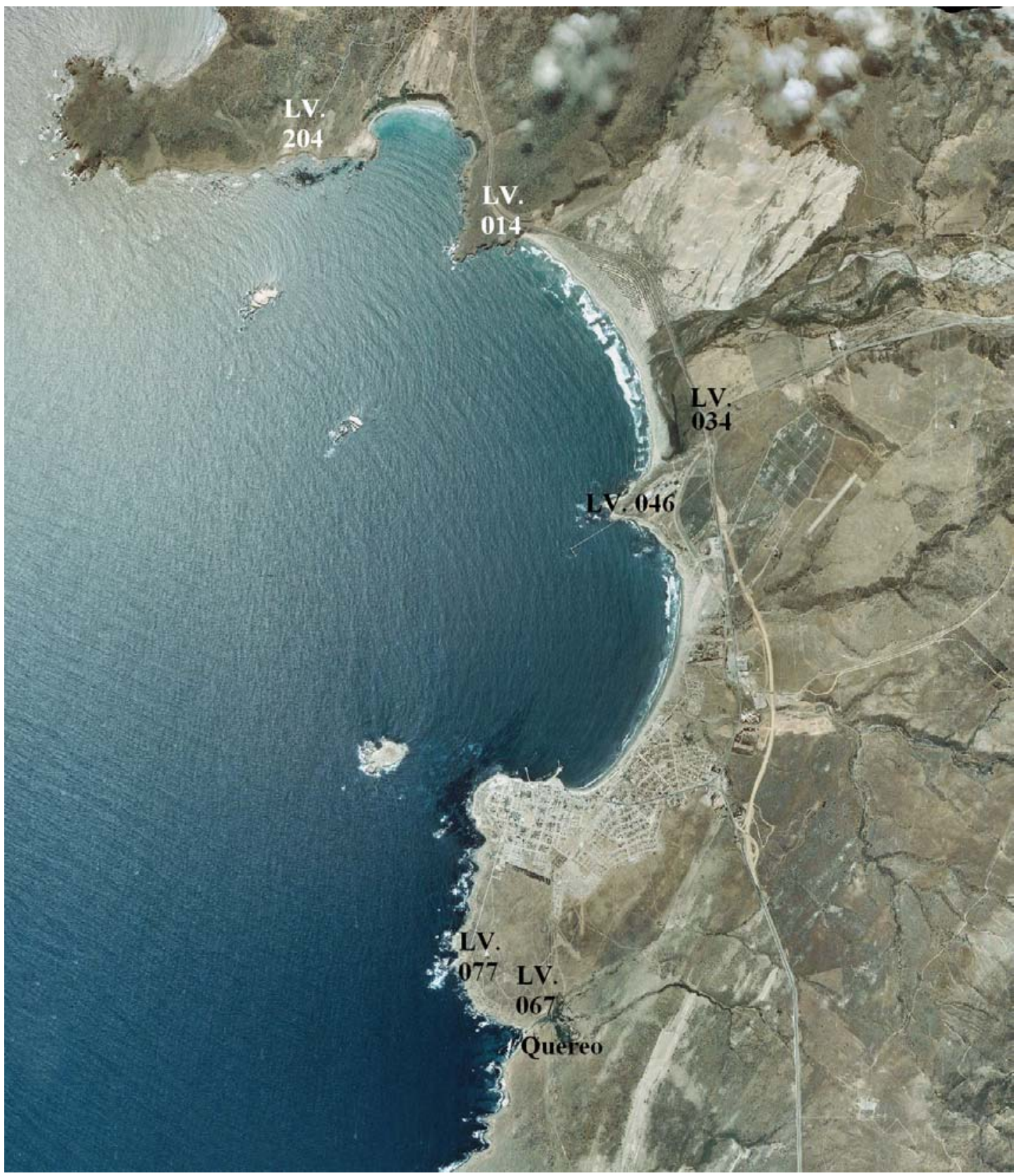

Figura 2. Área de estudio y asentamientos del Holoceno tardío. Study area and late Holocene settlements discussed in text.

Su potencia estratigráfica (entre 30 y $40 \mathrm{~cm}$ ), en conjunto con la variedad en la explotación de invertebrados y las características de su industria tecnológica, aluden a una funcionalidad residencial, más alejada del litoral. Se planteó que el asentamiento sería asignable al Complejo Cultural Papudo, característico del Holoceno medio, aunque en tiempos más tardíos (Barrera y Belmar 2000).
No obstante, las fechas que en la actualidad se manejan para el resto de asentamientos en la zona (Tabla 1) y sus similitudes contextuales permiten integrarlo al conjunto de yacimientos evaluados en el presente trabajo. Un fragmento de piedra horadada en el sitio, ergología característica de la unidad cultural Papudo, no niega lo anterior, en vistas que, localmente, entre los cazadores recolectores 
de ambos períodos es probable una continuidad poblacional (Méndez 2003).

Además de Penitente, otros yacimientos que exhiben este patrón de acumulación de basuras desordenadas, matrices carbonosas y altamente orgánicas, ecofactos diversos y fragmentados, con tecnología de bajo nivel de inversión laboral y apropiación de recursos pétreos inmediatos, son aquellos de Punta Chungo (LV. 046a y LV. 046b). Ello no debe pasarse por alto, en vistas que sobre la base de esta marcada similitud se cimienta la visión de un panorama integrado, en donde los asentamientos residenciales del Holoceno tardío exponen un patrón conductual generalizado. Punta Chungo A (LV. 046a, $22 \mathrm{msm}$ ) constituye un yacimiento arqueológico, conformado por tres montículos de basurales de valvas (Figura 3), de los cuales sólo uno fue intervenido estratigráficamente $\left(4 \mathrm{~m}^{2}\right.$, Jackson et al. 2004). En sus 3,4 m de estratigrafía, exhibe una importante reiteración en el uso del emplazamiento por grupos del Holoceno medio, a modo de campamento logístico de desconche. Por su parte, las variables contextuales características del evento ocupacional tardío (superpuesto a los anteriores) muestran: diversidad malacológica, sedimentos orgánicos, altas tasas de fragmentación (pisoteo), industrias líticas expeditivas caracterizadas por instrumentos sobre núcleos, lo que permite observar una diferente lógica funcional del campamento. Las disimilitudes se acentúan, en tanto la ocupación del Holoceno tardío exhibe una menor selectividad de las dimensiones de los locos (Concholepas concholepas); a lo que se suma la mayor variabilidad en los taxones consumidos, ya sean de fauna malacológica o de vertebrados (cánidos, mamíferos terrestres no identificados y aves, Jackson et al. 2004).

Una descripción casi idéntica podría ser sostenida para Punta Chungo B (LV. 046b). Este asentamiento, muy próximo al anterior $(400 \mathrm{~m})$, se emplaza sobre un sistema de dunas y corresponde a un gran montículo de conchal con escasas evidencias superficiales (Jackson y Méndez 2004). Una intervención de 6 unidades de excavación $\left(18 \mathrm{~m}^{2}\right)$

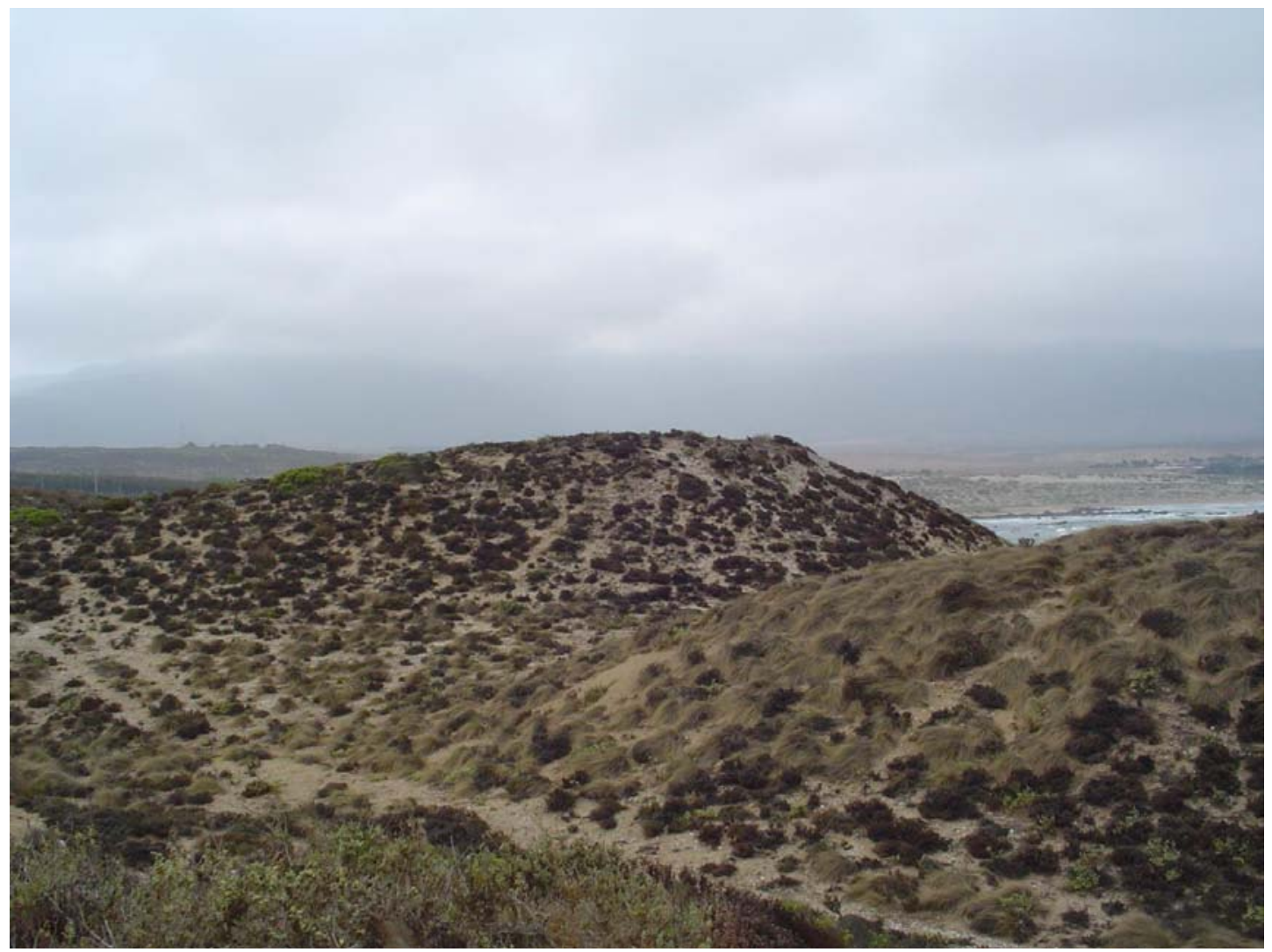

Figura 3. Basurales conchíferos monticulares del sitio de Punta Chungo (LV. 046a). Shell midden mounds at the Punta Chungo site (LV. 046a). 
reveló una secuencia ocupacional muy similar a la del yacimiento anterior, exponiendo el consecuente cambio radical en la funcionalidad del emplazamiento luego del advenimiento del Holoceno tardío. Las evidencias ecofactuales exhiben el mayor nivel de diversidad aún observado para estos contextos en el área de estudio, entre los que se cuenta una gran variedad de moluscos (muy fragmentados), restos de peces, ungulados, cánidos, mustélidos, roedores, marsupiales, aves, anuros y reptiles (lagartijas). Asimismo, destaca el registro de dos artefactos elaborados sobre hueso (punzón y cuenta tubular). Por su parte, las evidencias líticas revelaron un procuramiento en fuentes de materias primas cercanas, especialmente relacionadas al aprovechamiento de guijarros ovoidales. El instrumental exhibió guijarros con y sin modificaciones intencionales, instrumental sobre núcleos y sobre derivados (astillados y filos vivos). Finalmente, se identificó un premolar humano, pieza con intensiva abrasión producto de una dieta dura.

Un sexto contexto de interés, aunque sin datación absoluta, constituye el segundo depósito del sitio Los Cerrillos (LV. 077). Dispuesto sobre una ocupación fechada entre los 6.760 y 6.470 años cal. a.p. (Holoceno medio), es contextualmente homologable a aquellos descritos arriba: "gran variedad de moluscos, restos óseos y artefactos, muy entremezclados y triturados, en una matriz arenosa de color café ligeramente oscuro y poco compactada" (Jackson y Ampuero 1993:190). El sitio se emplaza sobre un paleoislote de la terraza II a 25 msm, próximo al intermareal rocoso. Se llevó a cabo una excavación de $14 \mathrm{~m}^{2}$ (dos campañas), registrándose el nivel tardío sólo en dos unidades $\left(8 \mathrm{~m}^{2}\right)$. El contexto asignable al Holoceno tardío expone tecnología expeditiva, caracterizada por instrumental sobre núcleos (de astillamiento uni y bilateral) y sobre guijarros con modificaciones. En cuanto a los taxones consumidos, además de los moluscos, destacan aves, roedores, mustélidos, félidos y cánidos.

Otro yacimiento necesario de mencionar es Quereo Norte (LV. 067), ya que, no obstante el contexto posee dos fechados AMS que lo asignan al lapso temporal discutido (Tabla 1), gran parte de los materiales culturales registrados son atribuibles a tecnología propia del Holoceno temprano (ca. 12.400 a 9.400 años cal. a.p.). El análisis conducido (Prado 2000) permite clasificarlo como un campamento de destazamiento inicial de presas, categoría funcional propia de las ocupaciones Huentelauquén (Arcaico temprano) del área de estudio (Jackson et al. 1999; Méndez 2002a). Una ocupación restringida estratigráficamente, atribuible a momentos tardíos, se superpone a la descrita. La acción de roedores fosoriales habría permitido el paso de materiales orgánicos de un estrato a otro; y no obstante se pretendió fechar el estrato basal, se obtuvo una cronología absoluta afín a la segunda ocupación. Integrar ambas dataciones (Tabla 1) a la discusión de este modelo es cuestionable, aun cuando sean coherentes entre sí y obtenidas con metodologías de alta precisión. Esta coyuntura alude a pensar que el sitio expondría una situación de disturbación fáunica (Schiffer 1996) o bien algún nivel de palimpsesto, que, a la luz de las evidencias, no permitiría una estricta discriminación entre sus ocupaciones.

Finalmente, el último campamento discutido constituye la Quebrada de Quereo (LV. 066), en específico aquel contexto en los primeros niveles del Miembro 6, Quereo III (Núñez 1983; Núñez et al. 1983; 1994). El yacimiento se ubica en un estrecho corredor tectónico que conforma el emplazamiento físico de un bosque relictual húmedo, a escasos metros del mar. El nivel cultural Quereo III corresponde a un basural de valvas de moluscos, que impone una diferente lógica de aprovechamiento del emplazamiento, en especial en relación a las ocupaciones finipleistocénicas. En este nivel cultural holocénico tardío se identificó una industria principalmente constituida por el aprovechamiento de guijarros. En cuanto a la subsistencia, destaca el consumo de otáridos y

Llama la atención (...) la práctica ausencia de bivalvos, pelecípedos, peces y fauna terrestre. Es decir, no hay restos de explotación de playas arenosas, mientras que (...) habla a favor de prácticas de recolección de mariscos de rocas, en el ámbito de la desembocadura (Núñez 1983:125).

Aún así, las evidencias hablan de un "régimen de alimentación marino (proteico), suficientemente diversificado" (Núñez et al. 1994:114).

En el área de estudio, el fin de estas manifestaciones podría ser preliminarmente fechado hacia momentos previos al 1.780 años a.p., datación obtenida a partir de un fragmento de cerámica Molle del sitio temprano Fundo de Agua Amarilla 
(LV. 099) (Jackson y Rodríguez 1998; Jackson et al. 1995; Seguel et al. 1995). No obstante, nos parece poco plausible un reemplazo poblacional brusco, en donde la sola presencia de tecnología cerámica justifique proponer la erradicación de una población local de profunda tradición cazadora recolectora. Ello se transforma en un argumento más sólido al considerar el escaso hiato temporal entre esta datación y aquella del contexto tardío de Punta Penitente (Tabla 1, Méndez 2002b).

\section{Caracterización Conductual e Indicadores Arqueológicos}

En conjunto, los asentamientos estudiados para este lapso temporal son más abundantes que en períodos anteriores. Por todas las evidencias expuestas, parecen responder a una distinta lógica de apropiación del espacio, en donde una población se estableció localmente (aunque siempre mantuvo su movilidad), aprovechando de manera más intensiva el medio costero de Los Vilos. Los contextos datados para estos momentos poseen un rango consistente entre los 4.000 y 2.000 años cal. a.p. y representan un uso significativo de las puntillas costeras (LV. 014, LV. 046a y b) y otros espacios de la segunda terraza marina (LV. 204 y LV. 077), algunos sectores más retirados del intermareal (LV. 034), posiblemente vinculados a la explotación de ambientes estuarinos y sistemas de quebradas y/o desembocaduras (Quereo, LV. 066 y LV. 067). Esta nueva lógica de asentamiento y movilidad habría estado caracterizada por un régimen de apropiación de un espectro amplio de recursos de disponibilidad inmediata. La movilidad residencial habría estado fundamentada en la selección de emplazamientos, en función de la distribución de los recursos costeros recolectables, de los cuales, los invertebrados sólo constituyen el conjunto de basuras de mayor visibilidad (prospecciones al interior confirman una mayor intensidad de uso de los espacios costeros). Desde esta perspectiva, el desarrollo de tecnologías aptas para soluciones inmediatas, habría conjugado un amplio conocimiento de la distribución local de las materias primas, en conjunto con altas tasas de descarte. Finalmente, se plantea que el proceso de recolección y el desconche de moluscos fue una labor colectiva, ejecutada por unidades más amplias que una reducida partida de especialistas logísticos.
El modelo interpretativo de movilidad residencial (Binford 1980) se fundamenta sobre ciertos indicadores conductuales derivados de la teoría arqueológica, entre ellos, primordialmente la proliferación de asentamientos, en conjunto con una manifiesta indiferenciación funcional entre éstos. En otras palabras, en los campamentos de los cazadores recolectores insertos en este tipo de movilidad, la preparación, procesamiento y consumo de toda la comida se esperaría que se localizaran en el sitio mismo, pudiendo observarse la completa materialidad de las actividades implicadas en ello; e incluso, a manera de expectativa, estas evidencias debieran tener un estrecho vínculo con la estructura ambiental inmediata (Chatters 1987). Ya que las poblaciones se mueven hacia los recursos, estas expectativas son especialmente manifiestas en la costa, puesto que, particularmente los moluscos, se encuentran con ubicuidad.

Uno de los indicadores segregados radica en la disposición espacial del asentamiento. Este proceso selectivo se manifiesta en el uso reiterado de un mismo espacio, expectativa que para las actividades de desconche, según Waselkov (1987), cumplirían los lugares en posición elevada en relación al resto del área general (terrazas marinas). En la zona de estudio es posible constatar este patrón, aunque sin descartar cierto nivel de flexibilidad y aprovechamiento de distintos espacios. Hipotéticamente, resulta interesante atribuir dicha tendencia a la necesidad de ubicar marcadores espaciales para la reunión poblacional postextracción de recursos, elemento facilitado por las puntillas costeras, las que además proveerían protección ante las inclemencias del viento, a fin de mantener campamentos estacionales por algunos días.

Uno de los argumentos más sustantivos que permiten plantear un sistema de movilidad residencial en el borde litoral es la mayor permanencia de contingentes poblacionales en los campamentos, a diferencia, por ejemplo, de los contextos logísticos (estos últimos se articulan funcionalmente con campamentos habitacionales cercanos a tres kilómetros al interior y son característicos del asentamiento en el Holoceno medio, Jackson 2002). Distintos indicadores arqueológicos han sido observados para su evaluación, entre los que destaca la significativa tasa de acumulación de basuras. Particularmente diagnóstico constituye el espesor de los depósitos de basurales, entre 40 y 60 cm (LV. 014: 50 cm, LV. 034: 33 a 40 cm, LV. 046a: 
$70 \mathrm{~cm}$, LV. 046b: 45 a $58 \mathrm{~cm}$, LV. 077: 60 a 65 $\mathrm{cm})$, los que no permiten diferenciar pisos ocupacionales. Estos depósitos parecen responder a la integración entre una fuerte actividad de desconche y la reiteración del uso de un mismo emplazamiento.

Otro de los indicadores clave de mayor permanencia en los campamentos corresponde a la cantidad de rocas despedazadas, producto de la acción del fuego, generalmente empleadas a manera de radiadores de calor para procesamiento de alimentos (Chatters 1987) o como límites de las estructuras de combustión. Particularmente significativa resulta la presencia de esta variable contextual en algunos de los yacimientos estudiados (LV. 014, LV. 034, LV. 046b). No obstante lo señalado, la presencia de mayor cantidad de personas en los asentamientos debiera traducirse en procesos destructivos que no permitirían observar los rasgos específicos conformados durante su ocupación (Chatters 1987). Este indicador se registra localmente en la escasa integridad de los fogones, cuya disrupción da pie a matrices carbonosas generalizadas, como las observadas en los contextos discutidos (LV. 014, LV. 034, LV. 046a y b, LV. 077). Estos depósitos estratigráficos estarían igualmente caracterizados por significativos contenidos orgánicos producto de la actividad de procesamiento de presas y recursos recolectados. Consecuente con lo anterior, otro indicador arqueológico que otorga fuerza a la interpretación, consiste en los importantes niveles de pisoteo de las evidencias. La manifestación más clara de esta conducta se registra en la significativa fragmentación de los moluscos (Claassen 1998), crustáceos, y en particular, los equinodermos (Méndez 2002a). Los contextos con estas características (LV. 014, LV. 046a y b, LV. 077, LV. 067 y Quereo) permiten al menos insinuar la posibilidad que los densos basurales significativamente pisoteados hayan sido producto de la actividad de unidades grupales completas conviviendo en un mismo espacio.

Sin lugar a dudas, uno de los elementos fundamentales en la caracterización de la movilidad residencial es la selección del área habitacional en función de la distribución de recursos. Bajo esta premisa, se podría esperar que los contextos exhibiesen algún grado de correspondencia entre el agregado natural inmediato al asentamiento y aquel recolectado por las poblaciones. Con el propósito de evaluar lo sostenido, se implementó una prueba de índice de similitud ${ }^{1}$ (Styles 1985) entre los contextos estudiados. De corresponder a una lógica de apropiación en medios inmediatamente próximos, los asentamientos debieran ser escasamente parecidos el uno del otro. Para ello se llevaron a cabo tantas operaciones como fueran necesarias $(n=15)$ para vincular, entre sí, a cada uno de los sitios (LV. 204, LV. 014, LV. 034, LV. 046a y b, y Quereo, sólo contextos datados). Los resultados exhiben una tendencia sustantivamente baja (índice de similitud promedio de 0,279, ds: 0,1), que corrobora la escasa similitud entre los contextos. Si bien se sostiene que, para este lapso temporal, las poblaciones ejercieron un aprovechamiento de la totalidad de la fauna del intermareal, sin lugar a dudas prevaleció una selectividad vinculada a aquello inmediatamente disponible en las inmediaciones de los campamentos. Esta idea permite sustentar que la movilidad fuera residencial, e incluso más, que dicha decisión se supeditara a estrategias de subsistencia predictivas, que anticiparan la presencia de los moluscos y otros recursos. El hecho que algunos sitios hayan mostrado mayor semejanza (similitud sobre 0.4, entre LV. 014, LV. 046a y b), e incluso mayor variabilidad específica, se atribuye a su proximidad ( $2 \mathrm{~km}$ aproximadamente y en la misma ensenada, Agua Amarilla).

Otra de las líneas argumentativas que han permitido generar este modelo radica en la evaluación de la tecnología desarrollada por estas poblaciones. Por una parte, la proliferación de implementos de molienda en los contextos del Holoceno tardío (LV. 204, LV. 014, LV. 034, LV 046a y b, LV. 077, Quereo) permite pensar en la realización de actividades propias de ámbitos habitacionales. Si bien se reconoce la existencia de tecnología de molienda en momentos anteriores, ésta no se registra tan difundida en todos los asentamientos. Por otra parte, la mencionada expeditividad del instrumental lítico (LV. 204, LV. 014, LV. 034, LV 046a y b, LV. 077, Quereo) se manifestaría en el bajo énfasis en los diseños e inversión energética en la confección. Ello se explicaría, según Chatters (1987), en relación a la necesidad, tanto de mantener un nivel de eficiencia en la apropiación de un amplio espectro como la necesidad de minimizar los costos de transporte entre bases residenciales. Registrar, como tendencia, las cadenas operativas completas en los campamentos, corrobora la apreciación y permite establecer que se esté llevando a cabo un amplio rango de actividades. Éstas pare- 
Tabla 2. Taxones malacológicos presentes en los contextos estudiados. Shell species present in the contexts examined.

\begin{tabular}{|c|c|c|c|c|c|c|c|}
\hline Taxones & LV.204 & LV.014 & LV.034 & LV.046a & LV.046b & LV.077 & Quereo \\
\hline Concholepas concholepas & $\mathrm{X}$ & $\mathrm{X}$ & $\mathrm{X}$ & $\mathrm{X}$ & $\mathrm{X}$ & $\mathrm{X}$ & $\mathrm{X}$ \\
\hline Mesodesma donacium & & $\mathrm{X}$ & $\mathrm{X}$ & $\mathrm{X}$ & $\mathrm{X}$ & $\mathrm{X}$ & $\mathrm{X}$ \\
\hline Tegula atra & $X$ & $X$ & $\mathrm{X}$ & $X$ & $\mathrm{X}$ & $\mathrm{X}$ & $\mathrm{X}$ \\
\hline Austromegabalanus psittacus & & $\mathrm{X}$ & & $\mathrm{X}$ & $\mathrm{X}$ & $\mathrm{X}$ & \\
\hline Loxechinus albus & $\mathrm{X}$ & $\mathrm{X}$ & & & & $\mathrm{X}$ & \\
\hline Acanthina monodon & & $\mathrm{X}$ & $\mathrm{X}$ & $X$ & $\mathrm{X}$ & $\mathrm{X}$ & \\
\hline Retrotapes rufa & & $X$ & $X$ & $X$ & $\mathrm{X}$ & $\mathrm{X}$ & \\
\hline Protothaca thaca & & $\mathrm{X}$ & & & $\mathrm{X}$ & & \\
\hline Perumytilus purpuratus & $\mathrm{X}$ & $\mathrm{X}$ & & $\mathrm{X}$ & $\mathrm{X}$ & $\mathrm{X}$ & \\
\hline Prisogaster niger & $\mathrm{X}$ & $\mathrm{X}$ & & $\mathrm{X}$ & $\mathrm{X}$ & $\mathrm{X}$ & \\
\hline Homalaspis plana & & $\mathrm{X}$ & & & $\mathrm{X}$ & $\mathrm{X}$ & \\
\hline Choromytilus chorus & & $\mathrm{X}$ & & $\mathrm{X}$ & $\mathrm{X}$ & & \\
\hline Fisurella nigra & & $\mathrm{X}$ & & $\mathrm{X}$ & $\mathrm{X}$ & $\mathrm{X}$ & \\
\hline Fisurella crassa & & $\mathrm{X}$ & & $\mathrm{X}$ & $\mathrm{X}$ & $\mathrm{X}$ & \\
\hline Fisurella picta & $\mathrm{X}$ & $\mathrm{X}$ & & $\mathrm{X}$ & $\mathrm{X}$ & & \\
\hline Fisurella latimarginata & & $\mathrm{X}$ & & $\mathrm{X}$ & $\mathrm{X}$ & $\mathrm{X}$ & \\
\hline Fisurella maxima & $\mathrm{X}$ & $\mathrm{X}$ & & $\mathrm{X}$ & $\mathrm{X}$ & & \\
\hline Fisurella costata & $\mathrm{X}$ & $\mathrm{X}$ & & $\mathrm{X}$ & $\mathrm{X}$ & & \\
\hline Fisurella sp. & & $\mathrm{X}$ & & $\mathrm{X}$ & & $X$ & $X$ \\
\hline Chiton granosus & & $\mathrm{X}$ & & $\mathrm{X}$ & & $\mathrm{X}$ & $\mathrm{X}$ \\
\hline Chiton magnificus bowenii & $\mathrm{X}$ & $\mathrm{X}$ & $\mathrm{X}$ & $\mathrm{X}$ & $\mathrm{X}$ & $\mathrm{X}$ & \\
\hline Acanthopleura echinata & $\mathrm{X}$ & $\mathrm{X}$ & $\mathrm{X}$ & $\mathrm{X}$ & $\mathrm{X}$ & $\mathrm{X}$ & \\
\hline Chiton sp. & $\mathrm{X}$ & $\mathrm{X}$ & & $\mathrm{X}$ & $\mathrm{X}$ & & $\mathrm{X}$ \\
\hline Diloma nigerina & $\mathrm{X}$ & & $\mathrm{X}$ & $\mathrm{X}$ & $\mathrm{X}$ & & \\
\hline Oliva peruvianna & & $\mathrm{X}$ & $\mathrm{X}$ & $\mathrm{X}$ & $\mathrm{X}$ & $\mathrm{X}$ & \\
\hline Tegula tridentata & $\mathrm{X}$ & & & $\mathrm{X}$ & $\mathrm{X}$ & $\mathrm{X}$ & \\
\hline Collisella sp. & & & & $X$ & & & \\
\hline
\end{tabular}

cen relacionadas al procesamiento de maderas y otros recursos perecibles, pero por sobre todo destaca gran cantidad de piezas líticas sin modificación, que hipotéticamente atribuimos a conductas de procesamiento de alimentos. Integralmente, se podría caracterizar a manera de una industria sobre núcleos y/o guijarros (Figura 4) vinculada a técnicas directas de astillamiento con percutores duros, la cual, bajo una perspectiva evolutiva, correspondería al modo de reavivado más simple y rápido, con mayor gasto de materias primas (Hayden 1989).

Sin lugar a dudas, uno de los principales argumentos para establecer la mencionada expeditividad es la discriminación de conductas de anticipación de la presencia de los recursos líticos al momento de su requerimiento (Nelson 1991). En este sentido, durante este lapso temporal el énfasis está puesto sobre los recursos de inmediato procuramiento, de calidad media baja y baja. El más claro ejemplo es el aprovechamiento del aflo- ramiento rocoso de diorita en Punta Penitente (sector $3 \mathrm{~A}$ de LV. 014), que vinculamos a este momento cronoestratigráfico (Méndez 2002a). Es esperable que en las ocupaciones residenciales la variabilidad de herramientas tenga una estrecha ligazón con aquellos recursos disponibles en el medio (Chatters 1987). Bastaría entonces recordar que para cada uno de los asentamientos descritos, sus excavadores siempre aluden, explícita o implícitamente, a estrategias de aprovisionamiento inmediatas (i.e. playas de guijarros adyacentes a los campamentos: LV. 204, LV. 014, LV. 034, LV. 046a y b, LV. 077, y Quereo). Sólo se podría establecer una mayor amplitud de rango de acción a partir de la presencia de cuarzo del área de Caimanes (40 km hacia el interior) y la ocurrencia eventual de instrumental bifacial manufacturado en sílices de posible origen más lejano. Respecto a este, es posible destacar las puntas de proyectil de morfología triangular o ligeramente lanceoladas (LV. 204, LV. 014, LV. 


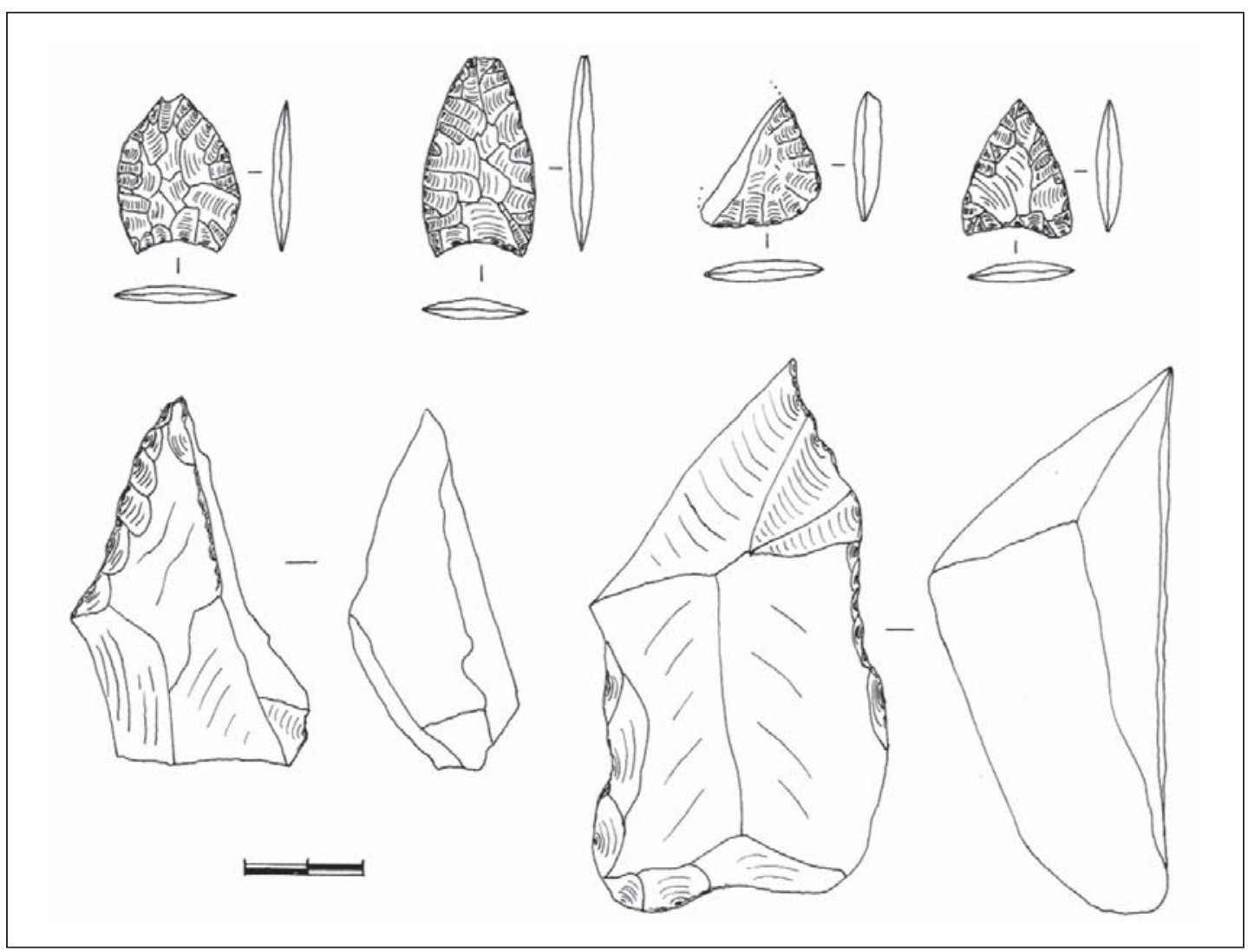

Figura 4. Material lítico característico de los contextos del Holoceno tardío. Lithic material typical of late Holocene contexts.

046a y b, LV. 077, LV. 067 y Quereo; Figura 4), que, aunque no exclusivas, son diagnósticas de estos grupos. La selección prioritaria de materias primas locales para su confección, confirma la idea de una movilidad en torno al eje litoral.

\section{Discusión}

En la comuna de Los Vilos, hacia la primera mitad del Holoceno tardío ( 4.000 a 2.000 años cal. a.p.), los asentamientos litorales proliferaron. Se registra invadida toda la diversidad de emplazamientos a lo largo de la costa, generándose depósitos arqueológicos de características sustantivamente homogéneas. Estos contextos, se observan superpuestos a casi todas las ocupaciones de momentos anteriores, Paleoindias (Quereo III), del Arcaico temprano: Huentelauquén (LV. 014, LV 067), del Arcaico medio: Papudo (LV. 014, LV. 046a y b, LV. 077), y también se ubican en emplazamientos sin previo uso (LV. 204, LV. 034). Este incremento y características particulares de los contextos se vinculan, hipotéticamente, a la implementación de una nueva lógica de movilidad de tipo residencial, centrada en el litoral y asociada a una mayor dependencia de los recursos costeros y terrestres adyacentes. Contrasta especialmente con la movilidad desarrollada durante el Holoceno medio, cuya aproximación al litoral fue sólo estacional (estival) y con un patrón de asentamientos que incluyó campamentos base y otros periféricos para la obtención de recursos específicos (Jackson 2002). A través de estas líneas se ha propuesto que las poblaciones locales del Holoceno tardío generaron un sistema de apropiación de alimentos bajo una base diaria $\mathrm{o}$, al menos, en cortos intervalos temporales. Casos etnográficos tienden a corroborar situaciones semejantes para grupos forrajeros, en donde cerca del $60 \%$ de los días del año se registra exclusivamente actividad (aunque sólo un segmento del grupo) de recolección de moluscos (Meehan 1982). 
La movilidad, y en particular el patrón de asentamientos sostenido anteriormente (Jackson et al. 1995), asignaba una funcionalidad logística a estos campamentos, relacionándolos a la explotación del intermareal y conductas de desconche local. No obstante, pensamos, ante el cúmulo de información, expectativas e indicadores teóricos esbozados, que estos campamentos constituirían más bien bases residenciales de corta duración, insertas en una lógica de apropiación sostenida de los recursos del medio litoral, en una lógica de movimientos latitudinales. Estos sitios podrían ser entendidos como bases residenciales autosuficientes, a las que se retorna a diario, siendo su clave la explotación de distintos microambientes a lo largo del ciclo anual, manteniendo la acumulación "excedentaria" al mínimo (Lee 1977). Si bien una de las características frecuentemente aludidas a la movilidad residencial, o grupos forrajeros, es la escasa probabilidad que los campamentos sean reocupados (Politis 1996), en la costa esta situación teóricamente tendería a verse modificada, en vistas de la concentración de los recursos en el área intermareal, destacándose la ocupación de las puntillas costeras. No obstante, los contextos estudiados no reflejan reocupaciones segregadas estratigráficamente, sino situaciones de palimpsesto, posiblemente en función de la recurrencia de este proceso. Esta situación bien podría observarse para los grupos que poblaron la localidad costera de Los Vilos en tiempos posteriores al 3.800 años a.p., los que dispusieron fundamentalmente su ordenamiento residencial latitudinal, aunque sin negar eventuales accesos $\mathrm{a}$ áreas del interior. En esta perspectiva, los recursos recolectables, vegetales y acuáticos (marinos y de estuario), podrían concebirse de mayor jerarquía que las presas cazadas en la ubicación de los campamentos. La recolección permitiría el procuramiento de una base subsistenciaria más bien mediatizada por la rutina y caracterizada por una alta confiabilidad. Sistemas "generalizadores" como estos tienden a incrementar la amplitud de la dieta a través de la inclusión de diferentes recursos, $y$, aunque su dimensión territorial sea restringida, ante la falta de competencia, su potencial de conquista de nuevos emplazamientos es alto (Dunnell 1994).
El conjunto de actividades de subsistencia (caza, recolección, obtención de moluscos, procuramiento de agua y minerales como la sal, plantas medicinales, entre otras) se mantendrían a manera de un esfuerzo constante a lo largo del año. Se puede interpretar lo anterior bajo un panorama de recolección en manos de las unidades domésticas, las cuales deciden ordenar su espacio social hacia los recursos, con el propósito de reducir sus costos de transporte. Se esperaría, bajo esta óptica, que todos los segmentos de estas sociedades coexistieran la mayor parte del tiempo, habiéndose reducido sustancialmente los grupos especializados. Es decir, resulta factible pensar que todas las agrupaciones etarias y genéricas del núcleo se integrasen en las actividades de obtención diaria del alimento.

\section{Vínculos Culturales}

De acuerdo a la tradición imperante en los estudios arqueológicos nacionales, las poblaciones de cazadores recolectores del Holoceno tardío en el Norte Semiárido $\left(30^{\circ}-32^{\circ} \mathrm{S}\right)$ han sido asumidas a entidades histórico culturales como Guanaqueros o Punta Teatinos (Schiappacasse y Niemeyer 1986). Lo anterior se sustenta sobre un cúmulo de estudios llevados a cabo en áreas más septentrionales dentro de la región de Coquimbo, en donde se manejan contextos claros y un conjunto de dataciones absolutas, que han permitido la propuesta cronocultural (Schiappacasse y Niemeyer 1986). No obstante, asumir lo anterior encuentra basamento exclusivamente en una asimilación ambiental, la cual vincula desarrollos culturales sólo por disponerse de manera contigua en un amplio territorio (Méndez 2003). La pregunta entonces radica en cuál es aquella población de recolectores locales de movilidad residencial en el litoral de Los Vilos, y si es factible considerar un vínculo cultural fundado en dicha proximidad espacial.

La segunda parte de la pregunta posee una resolución más evidente. Como primer punto, no han sido registrados parentescos ergológicos o tecnológicos que permitan relacionar las evidencias de Los Vilos con aquellas más septentrionales. A este respecto, la materialidad de la fase Guana- 
queros se observa más afín a desarrollos nortinos como la Cultura del Anzuelo de Concha. Asimismo, en el área meridional del Norte Semiárido no se han registrado indicadores como los patrones funerarios que caracterizan a la fase Punta Teatinos, o bien, Guanaqueros. También es necesario mencionar (y ponderar) que las investigaciones del área más al norte no han tomado en cuenta las variables contextuales y conductuales consideradas por nosotros.

Una segunda línea argumentativa que diferencia al área septentrional y meridional del Norte Semiárido, radica en sus tendencias cronológicas. Bastaría comparar las dataciones absolutas locales y las propuestas histórico culturales para el Arcaico tardío del Norte Semiárido (Schiappacasse y Niemeyer 1986; Kuzmanic y Castillo 1986). Esta periodificación establece las fases Guanaqueros (III), Punta Teatinos (IV) y Quebrada Honda (V), en lapsos temporales promediados del orden de los $4.000,3.500$ y 2.000 años a.p., respectivamente (Schiappacasse y Niemeyer 1986). En este sentido, si bien las fechas de Los Vilos son, parcialmente, contemporáneas con aquellas más septentrionales, las tendencias temporales ilustran una significativa diferencia (Méndez 2003). Las asignaciones cronológicas presentan al menos dos importantes agrupaciones (tendencias). En primer lugar, las asignaciones cronológicas -fases- vinculadas al Arcaico tardío del norte de la IV Región se definirían principalmente a partir de ergología y complejos funerarios, posicionándose desde momentos terminales del Holoceno medio y su transición al tardío. En segundo, se observa un segmento geográficamente acotado a la localidad de Los Vilos, fundado sobre similitudes contextuales, e interpretado como una lógica de asentamientos y movilidad pautada, imperante durante la primera mitad del Holoceno tardío. Los argumentos, hasta el momento, no permiten sostener continuidad entre un área y otra, sino más bien desarrollos independientes. Por su parte, las dataciones cronológicas en Los Vilos poseen un mayor grado de coherencia interna, y se condicen integralmente con los desarrollos locales de cazadores recolectores inmediatamente anteriores.

Investigaciones conducidas en el área de Los Vilos han permitido el desarrollo de un modelo de movilidad y aprovechamiento de la costa para momentos del Holoceno medio (Jackson 2002). Este patrón de acceso pautado postula que poblaciones de la vertiente oriental de la cordillera de los Andes acudirían al litoral en la estación estival, a fin de complementar sus recursos (Jackson 2002). Una vez concluido el hipertermal, hipotéticamente, algunas agrupaciones humanas, haciendo uso de su flexibilidad oportunista, no habrían retornado a la vertiente oriental, una vez completado el ciclo anual (Méndez 2003). Sumado a lo anterior, el retorno hacia condiciones más húmedas y benignas, en donde prevalecería menor contraste entre estaciones, habría dado lugar a un aumento en la disponibilidad de recursos y, por tanto, un incremento en la confiabilidad hacia la recolección. Junto con ello, el medio ambiente en la zona cordillerana (ambas vertientes) también se habría regenerado, cuestión que bien pudo desincentivar las motivaciones iniciales que llevaron a los cazadores del interior a acceder estacionalmente a la costa. De lo esbozado, se desprende que los grupos humanos del Holoceno medio constituirían probablemente aquel sustrato poblacional que dio paso a la adaptación litoral que observamos en momentos posteriores (Méndez 2003). Esta aseveración posee un fuerte correlato tecnológico y contextual, específicamente en lo referido a las industrias expeditivas y los yacimientos estudiados.

Agradecimientos: Investigación financiada a través de los proyectos FONDECYT 91-0026, 1950327 y 1990699 . Se agradece a todas las personas que participaron en las labores de campo y laboratorio de los yacimientos aquí presentados, y especialmente a Diego Artigas, Mónica Barrera y Carolina Belmar por los manuscritos facilitados. Se extiende el agradecimiento al Dr. Lautaro Núñez y a tres revisores anónimos por sus valiosos comentarios. 


\section{Referencias Citadas}

Artigas, D

2003 Descifrando la superficie: recolección superficial e intervención estratigráfica en un sitio Arcaico de la costa de Los Vilos. Boletín de la Sociedad Chilena de Arqueología 35/36:9-27.

Barrera, M. y C. Belmar

2000 Rescate del sitio arqueológico $\mathrm{n}^{\circ} 18$ Paso Inferior Conchalí: Nuevas evidencias del Complejo Papudo en el Norte Semiárido. Valles Revista de Estudios Regionales, manuscrito en posesión del autor.

Bate, L.

1971 Material lítico: metodología de clasificación. Noticiario Mensual del Museo Nacional de Historia Natural 181182:3-24.

Binford, L.

1980 Willow smoke and dogs' tails: hunter-gatherer settlement systems and archaeological site formation. American Antiquity 45:4-20.

Borrero, L., N. Franco, F. Carballo y F. Martín

1998-9 Arqueología de Estancia Alice, Lago Argentino. Cuadernos del Instituto Nacional de Antropología y Pensamiento Latinoamericano 18:31-48.

Chatters, J.

1987 Hunter-gatherer adaptations and assemblage structure. Journal of Anthropological Archaeology 6:336-375.

Claassen, C.

1998 Shells. Press Syndicate of the University of Cambridge, Cambridge.

Donoso, $\mathrm{C}$.

1981 Investigación Forestal y Desarrollo. CONAF y ONU, Santiago.

Dunnell, R.

1994 Why is there a hunter-gatherer archaeology? En Arqueología de Cazadores-Recolectores. Límites, Casos y Aperturas, editado por J. Lanata y L. Borrero. Arqueología Contemporánea 5:7-15.

Falabella, F., M. Planella y A. Pollastri

1991 Análisis de oxígeno 18 en material malacológico de Chile Central. Actas del XI Congreso Nacional de Arqueología Chilena Vol I:105-121. Museo Nacional de Historia Natural, Santiago.

Feathers, J.

1996 Luminescence dating and modern human origins. Evolutionary Anthropology 5:25-35.

Fuenzalida, P.

1965 Geografía Económica de Chile. CORFO, Santiago.

Hayden, B.

1989 From the chopper to the celt: the evolution of resharpening techniques. En Time, Energy and Stone Tools, editado por R. Torrence, pp. 7-16. Cambridge University Press, Cambridge.

Jackson, D.

2002 Cazadores y Recolectores del Holoceno Medio del Norte Semiárido de Chile. Tesis para optar al grado de Magíster en Arqueología, Departamento de Antropología, Facultad de Ciencias Sociales, Universidad de Chile, Santiago.

Jackson, D. y G. Ampuero

1993 Tecnología y recursos explotados en un campamento Arcaico de Los Vilos, Provincia del Choapa. Actas del XII
Congreso Nacional de Arqueología Chilena, Vol. II:189-200.

Boletín del Museo Regional de la Araucanía 4, Temuco.

Jackson, D., P. Báez y J. Arata

2004 Composición de conchales, estrategias de subsistencia y cambios paleoambientales en un asentamiento arcaico, Norte Chico de Chile. Boletín de la Sociedad Chilena de Arqueología, en prensa.

Jackson, D., P. Báez, R. Seguel y J. Arata

1996 Campamento arcaico para la explotación intermareal: Significado del desconche local de moluscos. Valles Revista de Estudios Regionales 2:89-109.

Jackson, D., P. Báez y L. Vargas

1995 Secuencia ocupacional y adaptaciones durante el Arcaico en la Comuna de Los Vilos, Provincia de Choapa. Actas del XIII Congreso Nacional de Arqueología Chilena, Tomo I:99-114. Hombre y Desierto, Antofagasta.

Jackson, D. y C. Méndez

2004 Reocupando el espacio: historia de un asentamiento multicomponente, sus relaciones inter-sitios y los cambios paleoambientales de la costa del Choapa. Werken 5 , en prensa.

Jackson, D. y A. Rodríguez

1998 Ocupación del complejo El Molle en la costa de Los Vilos, Provincia de Choapa. Boletín de la Sociedad Chilena de Arqueología 26:19-21.

Jackson, D., R. Seguel y P. Báez

2001 Informe de avance $\mathrm{N}^{\circ} 2$ proyecto FONDECYT 1990699. Manuscrito conservado en la Biblioteca de CONICYT, Santiago.

Jackson, D., R. Seguel, P. Báez y X. Prieto

1998 Informe final proyecto FONDECYT 1950372. Manuscrito conservado en la Biblioteca de CONICYT, Santiago.

1999 Asentamientos y evidencias culturales del Complejo Cultural Huentelauquén en la comuna de Los Vilos, Provincia del Choapa. Anales del Museo de Historia Natural de Valparaíso 24:5-28.

Jerardino, A.

1995 Late Holocene neoglacial episodes in southern South America an southern Africa: a comparison. The Holocene 5:361-368.

Kuzmanic, I. y G. Castillo

1986 Estadio Arcaico en la costa del norte semiárido de Chile. Chungara 16-17:89-94.

Lee, $\mathrm{R}$.

1977 [1968] What hunters do for a living, or, hope to make out on scarce resources. En Man the Hunter, editado por R. Lee e I. De Vore, pp. 30-48. Aldine Publishing Company, Chicago.

Maldonado, A. y C. Villagrán

2002 Paleoenvironmental changes in the semiarid coast of Chile $\left(\sim 32^{\circ} \mathrm{S}\right)$ during the last $6200 \mathrm{cal}$ years inferred from a swamp-forest pollen record. Quaternary Research 58:130138.

Meehan, B.

1982 Shell Bed to Shell Midden. Australian Institute of Aboriginal Studies, Camberra.

Méndez, C.

2002a Tecnología, Subsistencia y Movilidad en Punta Penitente (LV.014). Un acercamiento hacia los Patrones Con- 
ductuales de los Grupos de Cazadores Recolectores en el Litoral del Norte Semiárido. Memoria para optar al título de Arqueólogo. Departamento de Antropología, Facultad de Ciencias Sociales, Universidad de Chile, Santiago.

2002b Cazadores recolectores costeros y sus contextos de tarea: Una visión desde el asentamiento holocénico temprano de Punta Penitente (LV. 014), Los Vilos, IV Región. Chungara Revista de Antropología Chilena 34:153-166.

2003 Orígenes del asentamiento holocénico tardío de cazadores recolectores en el litoral del Choapa: Ensayo para la generación de una hipótesis de investigación. Werken 4:43-58.

Nelson, M.

1991 The study of technological organization. En Archaeological Method and Theory, editado por M. Schiffer, Vol. 3:57-100. University of Arizona Press, Arizona.

Núñez, L.

1983 Paleoindio y Arcaico en Chile. Diversidad, Secuencia y Procesos. ENAH e INAH, Ciudad de México.

Núñez, L., J. Varela y R. Casamiquela 1983 Ocupación Paleoindio en Quereo. Universidad del Norte, Antofagasta.

Núñez, L., J. Varela, R. Casamiquela y C. Villagrán 1994 Reconstrucción multidisciplinaria de la ocupación prehistórica de Quereo, centro de Chile. Latin American Antiquity 5:99-118.

Ota, Y. y R. Paskoff

1993 Holocene deposits on the coast of North-Central Chile: radiocarbon ages and implications for coastal changes. Revista Geológica de Chile 20:25-32.

Politis, G.

1996 Moving to produce: Nukak mobility and settlement patterns in Amazonia. World Archaeology 27:494-511.

Prado, C.

2000 Acerca de la funcionalidad de un asentamiento Huentelauquén próximo a Quebrada de Quereo, Provincia de Choapa. Actas del XIV Congreso Nacional de Arqueología Chilena, Vol. I:519-552. Museo Regional de Atacama, Copiapó.

Román, A. y D. Jackson

1998 Dataciones por termoluminiscencia de rocas de fogones de asentamientos arcaicos, Provincia del Choapa. Chungara 30:41-48.

Nota

1 Índice de similitud $=\mathbf{c} /(\mathbf{a}+\mathbf{b})$ permite, a partir de la presencia y ausencia de taxones (a un nivel específico), un rango comparable de similitud, entre 0 (absoluta diferencia) y 1 (completa similitud). La fórmula establece a c como
Schiappacasse, V. y H. Niemeyer

1986 El Arcaico en el norte semiárido de Chile: Un comentario. Chungara 16-17:95-98.

Schiffer, M.

1996 [1987] Formation Processes of the Archaeological Record. University of Utah Press, Salt Lake City.

Seguel, R., D. Jackson, A. Rodríguez, X. Novoa, M. Henríquez, P. Báez y D. Jackson

1995 Rescate de un asentamiento Diaguita costero: Proposición de una estrategia de investigación y conservación. Informe de proyecto DIBAM - FAI 94107. Manuscrito en posesión de los autores.

Shott, M.

1996 An exegesis of the curation concept. Journal of Anthropological Research 52:259-280.

Stein, J., editora

1992 Deciphering a Shell Midden. Academic Press. San Diego.

Stuiver, M., P. Reimer y T. Brazuiunas

1998 High-precision radiocarbon age calibration for terrestrial and marine simples. Radiocarbon 40:1127-1151.

Styles, B.

1985 Reconstruction of availability and utilization of food resources. En The Analysis of Prehistoric Diets, editado por R. Gilberg y J. Mieike, pp. 21-59. Academic Press, New York.

Varela, J.

1981 Geología del Cuaternario del área de Los Vilos - Ensenada El Negro (IV Región) y su relación con la existencia del bosque relicto de Quebrada Quereo. Comunicaciones 33:17-30.

Villagrán, C. y J. Varela

1990 Palynological evidence for increased aridity on the central Chilean coast during the Holocene. Quaternary Research 34:198-207.

Waselkov, G.

1987 Shellfish gathering and shell midden archaeology. En Advances in Archaeological Method and Theory, editado por M. Schiffer, Vol 10:257-310. Academic Press, New York.

el total de especies compartidas entre dos unidades diferentes, y a y b como el número total de especies presentes individualmente en cada una de las unidades (Tabla 2). 
